\title{
Fortgeschrittene Kopf-Hals-Tumoren \\ Cetuximab hat sich im klinischen Alltag auf breiter Basis durchgesetzt
}

Der IgG1-Antikörpers Cetuximab (Erbitux ${ }^{\circledR}$ ) gilt heute in Kombination mit einer platinbasierten Chemotherapie als Standard für die Erstlinientherapie von rezidivierten und/oder metastasierten Kopf-Hals-Tumoren. Dies wurde im Rahmen eines interdisziplinären Satellitensymposiums beim ICHNO (3rd International Conference on Innovative Approaches in Head and Neck Oncology), der Anfang Februar in Barcelona, Spanien stattfand diskutiert.

Bei mehr als der Hälfte der Patienten mit Kopf-Hals-Tumoren treten nach der Behandlung des Primärtumors Rezidive oder Metastasen auf. Dazu kommt, dass die Erkrankung bei vielen Patienten schon bei der Erstdiagnose lokal fortgeschritten ist oder dass bereits Fernmetastasen vorliegen.

„Mit dem in der Phase-IIIStudie EXTREME (Erbitux in $1^{\text {st }}$-line Treatment of recurrent or metastatic head and neck cancer) getesteten Cetuximab-haltigen Behandlungsregime wurde nach 30 Jahren der Stagnation endlich ein entscheidender Fortschritt für die Erstlinientherapie von rezidivierten und/oder metastasierten Kopf-Hals-Tumoren erreicht“, erklärte Jan B. Vermorken, Antwerpen/Belgien. Die Addition des monoklonalen IgG1-Antikörpers zum bisherigen Therapiestandard Carboplatin/ Cisplatin plus 5-Fluorouracil (5-FU) führte zu folgenden Verbesserungen [Vermorken et al. N Engl J Med 2008; 359:1116-27]:

- Sie verlängerte das mediane Gesamtüberleben um fast drei Monate (10,1 vs. 7,4 Monate; $\mathrm{p}=0,04)$.

- Sie steigerte die Dauer des progressionsfreien Überlebens von 3,3 auf 5,6 Monate (relative $\mathrm{Ri}$ sikoreduktion $46 \%$; $<<0,001$ ).

- Und sie erhöhte die objektive Ansprechrate relativ um $80 \%(20$ vs. $36 \%$; p < 0,001).
Die Analyse der häufigsten Grad-3/4-Nebenwirkungen unter dem Cetuximab-Regime erbrachte keine unerwarteten Reaktionen; insbesondere war die Inzidenz schwerer Anämien, Neutropenien und Thrombozytopenien in den beiden Studienarmen nicht signifikant verschieden [Mesia $\mathrm{R}$ et al. Ann Onco12010;21:1967-73]. Die Lebensqualität wurde von den Patienten in beiden Behandlungsgruppen vergleichbar eingestuft.

„Diese positiven Ergebnisse haben dazu geführt, dass Cetuximab in Kombination mit einer platinhaltigen Chemotherapie bei nicht vorbehandelten Patienten mit rezidiviertem und/oder metastasiertem Kopf-Hals-Tumor mittlerweile der Therapiestandard ist", erklärte Vermorken. Diese Therapieoption wurde daher mit dem höchsten Empfehlungsgrad in die EHNS/ESMO-ESTRO-Leitlinien aufgenommen. $\quad d b$

Nach Information von Merck Serono

\section{Personalisierte Therapiekonzepte haben Erfolg NSCLC: EGFR-Status bei der Therapieentscheidung beachten}

\begin{abstract}
Personalisierte Therapiekonzepte, die individuelle biologische Merkmale berücksichtigen, haben Erfolg: „Der prädiktive Wert von Biomarkern wurde lange unterschätzt", so Manfred Dietel, Berlin, „Diagnostik und Prädiktion gilt es künftig jedoch zu kombinieren."
\end{abstract}

Wie effektiv dies ist, zeigt sich unter anderem an aktivierenden EGFR-Mutationen beim nichtkleinzeligen Lungenkarzinom (NSCLC). Für die etwa $10 \%$ der Patienten, deren Tumor diese Mutationen aufweist, sind EGFRTyrosinkinase-Hemmer wie Gefitinib (Iressa ${ }^{\circledR}$ ) sowohl hinsichtlich des Tumoransprechens als auch des progressionsfreien Überlebens eine erheblich wirksamere
Alternative zur konventionellen Kombinationschemotherapie [Dietel M, Schirmacher P. Onkologe. 2010;16:63-6].

Der bedarfsgerechte Einsatz von TKI ist laut Norbert Frickhofen, Wiesbaden, „ein echter gischen Therapie“. Entscheidend für den Therapieerfolg ist jedoch die Bestimmung des EGFR-Mutationsstatus: „Sie muss bei allen Quantensprung in der onkolo-
NSCLC-Patienten als verpflichtende prätherapeutische Testung durchgeführt werden“, ergänzte Dietel. Die routinemäßige $\mathrm{Mu}$ tationstestung sollte jedoch nur in zertifizierten Pathologieinstituten erfolgen. Hierzu haben die Deutsche Gesellschaft für Pathologie und der Bundesverband ein Zertifizierungsverfahren durch Ringversuche namens QuiP (Qualität in der Pathologie) initiiert.

\section{bf}

